\title{
MODOS ESPONTÁNEOS DE RAZONAR: UN ANÁLISIS DE SU INCIDENCIA SOBRE EL APRENDIZAJE DEL CONOCIMIENTO FÍSICO A NIVEL UNIVERSITARIO BÁSICO
}

SALINAS DE SANDOVAL, J., CUDMANI, L.C. DE y PESA DE DANÓN M. Instituto de Física. FCEYT. UNT. Av. Independencia, 1800. 4000 Tucumán, Argentina.

\begin{abstract}
SUMMARY
The acquisition of knowledge in different domains may share certain general ways of reasoning; but besides this, each specific domain may require specific ways of reasoning.

In this paper we identify and we characterize four manifestations of spontaneous ways of reasoning, and we analize up to what extent they act as obstacles for a meaningful learning of scientific knowledge about simple electric circuits. Finally, we suggest some general ways in which our results may be taken into account into order to improve basic physics teaching at the universities.
\end{abstract}

\section{INTRODUCCION. PLANTEO DEL PROBLEMA. OBJETIVOS DEL TRABAJO}

A través de la interacción con experiencias de la vida cotidiana, y con otros individuos, las personas elaboramos espontáneamente diversas concepciones sobre los fenómenos físicos. Este conocimiento, que generaImente nos basta para enfrentar las exigencias de todos los días, muchas veces no coincide con las teorías que actualmente acepta la comunidad científica para explicar los mismos fenómenos.

Sin embargo, diversas investigaciones han puesto al descubierto la existencia de un cierto paralelismo entre esos esquemas cognoscitivos espontáneos y construcciones conceptuales que fueron aceptadas en épocas pasadas por la comunidad cientifica (Piaget, 1972; Piaget y García, 1982; extensa bibliografía relacionada con las concepciones previas de los estudiantes).

En particular, la física espontánea, de sentido común, con la que muchos alumnos llegan a los ciclos básicos universitarios, parece estar vertebrada con criterios y modos de razonar que en muchos aspectos recuerdan los de metodologías superadas por la comunidad científica durante el proceso que dio origen a la física moderna clásica (Bachelard, 1972; Gil, 1983; Carrascosa y Gil, 1985).

El surgimiento de esta física moderna clásica significó, entre otras cosas, abandonar la pretensión de aprehender globaimente la naturaleza, extremadamente compleja, de los fenómenos naturales, y reemplazarla por el intento de comprender los comportamientos en situaciones modelizadas, obtenidas como abstracción e idealización de los casos reales (Bunge, 1985; Matthews, 1992).

La observación cualitativa no controlada deja lugar a la experimentación científica, cuantitativa, controlada, reproducible (Hodson, 1986).

Las certidumbres son reemplazadas por hipótesis que pueden ser tan audaces como se considere conveniente, pero que han de ser rigurosamente contrastadas (Popper, 1985). 
Todo se cuestiona. Nada está establecido para siempre. Y el espíritu sistemático se impone. Los conocimientos deben conformar un cuerpo que muestre la coherencia con otros resultados y otras situaciones. Las teorizaciones han de ser integradoras: la fragmentación en estudios específicos sólo cobra sentido cuando se sistematiza en una estructura conceptual globalizadora en la que las conclusiones se enriquecen y son controladas por otras conclusiones y, a la vez, ellas mismas enriquecen y controlan a otras conclusiones (Hempel, 1987).

El cambio conceptual de la física moderna clásica aparece acompañado de cambios en las valoraciones y en los objetivos, en los criterios y modos de razonar involucrados en la construcción y en la contrastación del conocimiento, en los ideales explicativos, etc. Aparece, en síntesis, como un tipo de saber cualitativamente diferente al que elaboramos intuitivamente (basándonos en el sentido común y en modos espontáneos de razonar).

A nivel de los ciclos básicos universitarios, la física contemporánea -cuando aparece-sólo se trata a nivel de información, de modo que en general los profesores no enfrentamos en este nivel el problema educativo de favorecer en los estudiantes los cambios vinculados a la transición desde teorías clásicas hacia ia relatividad, Ia cuántica, la termodinámica de procesos irreversibles, etc.

Desde una perspectiva constructivista, es posible hipotetizar que, así como diversos criterios metodológicos y modos de razonamiento espontáneo son inadecuados para los objetivos perseguidos con las teorías de la física moderna clásica, ellos serán también inadecuados para el aprendizaje de esas teorías en ciclos básicos universitarios (Gil y Carrascosa, 1985).

En este trabajo presentamos resultados empíricos, interpretaciones y justificaciones teoricas que a nuestro entender apoyan fuertemente esa hipótesis. Hemos centrado nuestro análisis en respuestas proporcionadas por estudiantes de ciclos básicos de carreras científicas a situaciones problemáticas referidas a circuitos eléctricos sencillos.

Cabe hipotetizar que los modos de razonamiento espontáneos que hemos detectado como obstáculo para un adecuado aprendizaje de esta área temática aparezcan también en otros contenidos donde intervengan procesos o sucesos físicos (y por tanto, conceptuaciones teóricas) de estructura análoga (conducción del calor, hidrodinámica).

\section{FORMULACIÓN DE LA HIPÓTESIS GENERAL Y DE LAS HIPOTESIS DERIVADAS}

La hipótesis general que ha sido esbozada en la introduccion fue enunciada de una manera mas precisa y fue operativizada a través de nuevas hipótesis, más detalladas y más directamente contrastables.
La formulación que presentamos en este trabajo de la hipótesis general y de las hipótesis derivadas fue pulida a través de ensayos piloto (cuestionarios, observaciones en clase, entrevistas, evaluaciones, etc.) y de consultas bibliográficas sobre los que informamos en otro trabajo (Salinas y Cudmani, 1993).

A través de una extensa práctica docente en un laboratorio de física básica para estudiantes de carreras de ingeniería y licenciatura en física, habíamos enfrentado reiteradamente situaciones que parecían mostrar que, si los estudiantes no razonan correctamente, ellos no comprenden correctamente los significados de las concep. ciones físicas.

Esos modos de razonar, a su vez, no parecían ser siempre los mismos, sino que parecían depender del contenido específico en cuestión.

Encaramos entonces una labor más sistemática. Decidimos centrar nuestro estudio en el campo de los circuitos eléctricos sencillos, donde habíamos detectado ya algunos comportamientos sobre los que parecía interesante profundizar. La búsqueda bibliográfica nos permitió conocer resultados e interpretaciones pertinentes (Viennot, 1983; Closset, 1983; Villani y Pacca, 1990; Manrique et al., 1989 , entre otros) y nos orientó para el diseño de experiencias piloto y para una observación más eficaz de los comportamientos en el aula.

Sobre la base de los resultados obtenidos con experiencias piloto, y de la realimentación permanente con la práctica docente, en un marco térico constructivista, hemos formulado las siguientes hipótesis de trabajo (Salinas y Cudmani, 1993):

\section{Hipótesis general}

H. a) «Los estudiantes de ciclos básicos de carreras, científicas emplean modos de razonamiento no científicos al enfrentar situaciones problemáticas relacionadas con el diseño y el funcionamiento de circuitos eléctricos simples."

b) «Esto los conduce a serias incomprensiones del conocimiento ff́sico involucrado.»

\section{Hipótesis derivadas}

H1. «Los estudiantes simplifican acríticamente los problemas, suponiendo a priori y sin control que la respuesta depende de una sola variable" (razonamiento monoconceptual).

H2. «Los estudiantes no consideran Ios efectos mutuos entre diversos elementos presentes en la situación y reducen su análisis a cambios locales o a deducciones directas» (razonamiento no sistemático, irreversible). 
H3. «Los estudiantes emplean los formulismos matemáticos y otros símbolos representacionales despojándolos de significado físico» (razonamiento puramente algorítmico).

H4. «Los estudiantes atienden más a las propiedades que a las funciones de los elementos en juego en la situación problemática, sean estos conceptuales ofácticos» (razonamiento reduccionista).

H5. «Las respuestas de los estudiantes basadas en los tipos de razonamiento no científico identificados muestran serias incomprensiones del conocimiento científico involucrado" (aprendizaje insatisfactorio).

Cabe hacer dos observaciones:

Para la situación planteada en la hipótesis H2. un marco piagetiano de interpretación sugeriría que esta imposibilidad de manejar una situación como sistema tiene como importante causa la no integración de las operaciones inversas. La irreversibilidad de las operaciones sería una condición necesaria para un tratamiento holístico; caso contrario, se razonaría en términos de hábitos irreversibles (Piaget, 1973). Téngase en cuenta que en este trabajo no se atribuye a esta reversibilidad o irreversibilidad de las operaciones un carácter absoluto ni independiente del contexto. Por el contrario, creemos que los razonamientos naturales son esencialmente dependientes de un contexto.

En cuanto a la hipotesis $\mathrm{H} 5$, puede ser útil señalar que las inconsistencias y falta de coherencia en las respuestas pueden aparecer como manifestaciones de mezclas acríticas de elementos espontáneos y científicos en el sistema cognoscitivo del aprendiz.

\section{METODOLOGÍA DE INVESTIGACIÓN}

La medicion fue realizada con una técnica de tratamiento múltiple: cada una de las hipótesis derivada fue controlada mediante diferentes cuestiones planteadas a los estudiantes.

Las repuestas de los estudiantes fueron recogidas a través de cuestionarios que debían ser respondidos por escrito.

Estos cuestionarios fueron administrados como parte de un proceso de evaluación continua implementada en un laboratorio de física básica universitaria para carreras de ingeniería y licenciatura en física (materia correspondiente al segundo año lectivo de las carreras).

Los estudiantes respondieron los cuestionarios a posteriori del tratamiento térico-experimental de los contenidos involucrados. Tenían libre acceso a libros de texto durante la elaboración de las respuestas. Para más detalles sobre la metodología de enseñanza empleada, puede consultarse Salinas y Cudmani (1993).
En el apéndice se presentan los enunciados empleados para contrastar las hipótesis de este trabajo. En general se trata de situaciones físicas muy simples (diseñadas en función de las hipótesis que guian la investigación), que solicitan que el estudiante formule un pronóstico justificado.

Una vez recogidos los cuestionarios con las respuestas de los alumnos, se los corrigió y devolvió corregidos en la próxima reunión de trabajo. A partir de este momento se sucedieron diversas actividades de entrevistas, consuitas, etc. con los estudiantes, a fin de realimentar el proceso de aprendizaje con los resultados obtenidos con la aplicación de los cuestionarios.

Este trabajo está centrado en el análisis e interpretación de las respuestas que los estudiantes brindaron por escrito, luego de haber realizado un estudio teórico-experimental de circuitos eléctricos simples y antes de participar en esas actividades de realimentación.

Los porcentajes obtenidos se manejan como cifras redondas.

En total intervinieron 77 estudiantes, todos ellos alumnos regulares de segundo año de ingeniería y licenciados en física. Su distribución para responder a las distintas cuestiones fue aleatorizada. En números redondos, cada cuestión fue administrada a unos 20 estudiantes, y cada estudiante respondió a unas dos cuestiones relacionadas con diferentes hipótesis o diferentes contenidos específicos.

La cifra « $\mathrm{N}=\ldots$... que aparece en cada una de las cuatro situaciones problemáticas del apéndice indica a cuantos alumnos fue administrada.

\section{ANÁLISIS E INTERPRETACIÓN DE LOS RESULTADOS}

\section{H1. Razonamiento monoconceptual}

Este tipo de razonamiento aparece en las respuestas de los estudiantes a las situaciones planteadas en los enunciados $1,2,3,4$ y 5 del apéndice.

En el problema núm. 1, de los 20 alumnos, sólo 7 responden correctamente y explican que, cuando crece

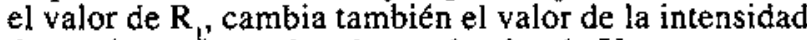
de corriente de modo tal que el valor de $\mathrm{V}$ aumenta.

De los 13 restantes, 6 afirman que el razonamiento es correcto "porque la caída de potencial es directamente proporcional al valor de la resistencia», y 4 aceptan el razonamiento «porque es una aplicación de la ley de Ohm». En ambos casos (que agrupados reúnen el 50\% de la muestra) subyace el supuesto (implícito o explícito) de que la intensidad de corriente es constante, comportamiento típico de un modo de razonar monowconceptual. 
En el problema núm. 2, de los 20 alumnos, sólo 4 responden correctamente, y concluyen que aumentará la intensidad luminosa emitida por el foco B.

De los 16 restantes, 4 señalan que, como los focos A y B están en paralelo con la fuente, la tensión sobre $B$ no se modifica por el hecho de desconectar $\mathrm{A}$, y deducen que la intensidad luminosa emitida por B no cambia cuando se abre el interruptor $S$, según la expresión $P o t=V^{2} / R$. No tienen en cuenta la influencia de la resistencia interna de la fuente; suponen incorrectamente que la diferencia de potencial entre los puntos 1 y 2 (ver figura I) es la misma antes y después de abrir el interruptor $S$.

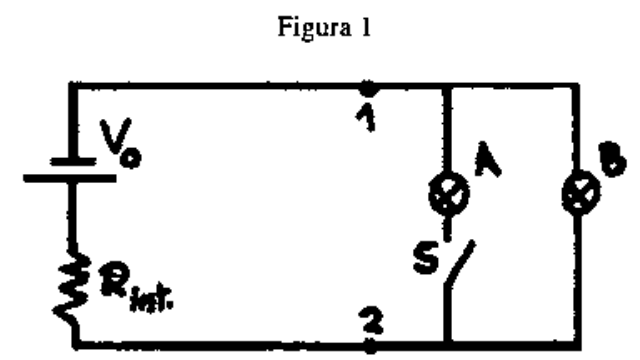

Otros 4 estudiantes calculan la resistencia equivalente del circuito antes y después de abrir el interruptor $S$, advierten que ésta aumenta al eliminar el foco $\mathrm{A}$, concluyen que «la corriente» es menor en el segundo caso que en el primero y deducen que el foco $B$ disminuirá su intensidad luminosa de acuerdo con la relación $P o t=I^{2}$. R. No tienen en cuenta la bifurcación de corrientes en la conexión en paralelo antes de abrir el interruptor S. En algunos casos, además no toman en cuenta la resistencia interna de la fuente al calcular la resistencia equivalente del circuito.

Finalmente, 3 estudiantes explican que al abrir el interruptor $\mathrm{S}$, la corriente que antes se dividía entre los puntos A y B ahora pasa íntegramente por B, y deducen que la intensidad luminosa emitida por $B$ aumenta, puesto que $P o t=I^{2}$.R. No tienen en cuenta que el valor de la intensidad de corriente total ha cambiado al modificarse el valor de la resistencia de carga conectada a la fuente.

Estos resultados dan un total de $55 \%$ de estudiantes con razonamiento monoconceptual en este problema.

En el problema núm. 3, de los 31 alumnos, sólo 8 responden correctamente y concluyen que la intensidad luminosa emitida por el foco $D$ disminuye, mientras aumentan las de los focos A y C.

De los 23 estudiantes restantes, 12 calculan la resistencia equivalente del circuito antes y después que se queme el foco $B$, advierten que ésta aumenta al eliminar el foco B del circuito, concluyen que la intensidad de la corriente eléctrica es menor en el segundo caso que en el primero y deducen que el foco $D$ disminuirá su intensidad luminosa. A continuación señalan que las intensidades luminosas de A y C no varían porque están conectadas en paralelo y la diferencia de potencial sobre ellos no cambia. No tienen en cuenta que la diferencia de potencial entre los puntos 1 y 2 (ver figura 2) depende de la resistencia equivalente del circuito, pues no consideran la tesistencia del foco $\mathrm{D}$, que está conectado a la fuente.

Figura 2
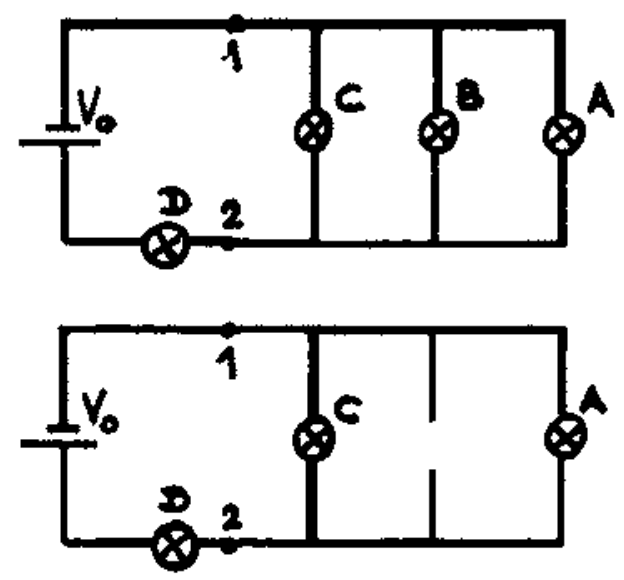

Otros 7 estudiantes señalan que la corriente que antes se dividía en tres partes (para atravesar los focos A, B y C), ahora tiene sólo dos caminos posibies (a través de los focos A y C); que, por lo tanto, aumenta la intensidad luminosa emitida por A y C, mientras permanece constante la de $\mathrm{D}$. No tienen en cuenta que, al quemarse e! foco $B$, ha cambiado la resistencia equivalente del circuito.

Suponen incorrectamente que la intensidad de corriente eléctrica es la misma antes y después de que se queme el foco $B$.

Estos resultados dan un total de más del $60 \%$ de estudiantes con razonamiento monoconceptual en este problema. Obsérvese la extraordinaria similitud en los modos de razonamiento subyacentes en las respuestas dadas a los problemas núm. 2 y núm. 3 , que fueron administrados a diferentes estudiantes.

En el problema núm. 4, de los 20 alumnos 11 , responden correctamente y afirman que la mínima intensidad luminosa se obtiene con el circuito III y la máxima con el I. y lo justifican correctamente.

De los 8 estudiantes restantes, 4 consideran que ia máxima intensidad luminosa se obtiene con el circuito III. Lo justifican calculando las resistencias equivalentes de los tres circuitos, mostrando que la del circuito III es la menor de todas, y concluyendo que «la corriente» es, por tanto, máxima para ese circuito. No tienen en cuenta la bifurcación de la corriente en la conexión en paralelo.

Con el mismo razonamiento, otros 4 alumnos concluyen que la minima intensidad luminosa se obtiene con el 
circuito $\mathrm{IL}$, que es el que posee Ia máxima resistencia equivalente.

Es decir, que el $40 \%$ de los estudiantes emplea razonamiento monoconceptual al responder a este problema.

En el problema núm. 5, de los 23 alumnos, 9 responden, correctamente, que disminuye la intensidad luminosa emitida por los focos A, E y F y que aumenta la del foco $D, y$ lo justifican correctamente.

El resto de los alumnos, que constituye el $60 \%$ de la muestra, pone de manifiesto razonamientos monoconceptuales. Tal es el caso cuando basan su razonamiento en la suposición de que el valor de la intensidad de corriente total no cambia al eliminar el foco B, o cuando no tienen en cuenta la bifurcación de la corriente en la conexion en paralelo, repitiendo modos de razonar que ya hemos analizado en las respuestas dadas a los problemas núm. 2 y núm. 3. Cabe señalar que, en los tres casos, (problemas núm. 2, 3 y 5), las muestras de estudiantes eran diferentes.

\section{H2. Razonamiento no sistémico, irreversible}

Este tipo de razonamiento aparece en las respuestas de los estudiantes a las situaciones planteadas en los enunciados $1,2,3,5,6$, y 7 del apéndice.

En Ios problemas núm. 1, núm. 2 y núm. 3, las respuestas de los estudiantes ponen de manifiesto este tipo de razonamiento cuando suponen que la intensidad de la corriente total (en una conexión en serie), o la caída de potencial (en las ramas de una conexión en paralelo), no cambian aun cuando haya modificaciones en alguna parte del circuito. Este tipo de comportamiento se observa en el $50 \%$ de los estudiantes en el problema núm. 1 , en el $35 \%$ de los estudiantes en el problema núm. 2 , y en el $60 \%$ de los estudiantes en el problema núm. 3 .

En el problema núm. 5 , el $40 \%$ de los estudiantes no toma en consideración el carácter sistémico del circuito, no tiene cuenta las interacciones mutuas entre los elementos del circuito y predice que las intensidades Iuminosas emitidas por los focos A, E y F no varían, mientras que la del foco $\mathrm{D}$ aumenta.

Parece interesante mencionar, como casos especiales, las respuestas de dos alumnos, que explícitamente distinguen el comportamiento de los focos que están «antes» de B y "después» de B, según un sentido de circulación que va desde el foco A hacia el foco F. Para ellos, la intensidad luminosa emitida por el foco A no cambia, pero sí lo hace la de los focos E y F. Este tipo de razonamiento ha sido identificado y caracterizado por distintos autores (Closset y otros, 1983), que le han asignado el nombre de «razonamiento secuencial».

En cuanto a los problemas núm. 6 y núm. 7 , el $70 \%$ de los estudiantes resuelve correctamente el problema núm. 6 , mientras que no llega al $25 \%$ el porcentaje de los que resuelven correctamente el problema núm. 7 (se trata de dos muestras diferentes de estudiantes). Podría aducirse que en ambos casos los estudiantes deben tener en cuenta la influencia convergente de distintos aspectos sobre el error cometido en la determinación del valor de la resistencia $R$. Pero, mientras la resolución del problema núm. 6 sólo requiere de la aplicación de un algoritmo de cálculo preestableciđo, el problema núm. 7 abre la posibilidad de varias respuestas correctas y exige tomar decisiones entre varias alternativas posibles, entre diferentes maneras de tomar en cuenta los factores que actúan e interactúan. Las dificultades observadas pueden relacionarse con resultados obtenidos en los estudios piagetianos sobre operaciones inversas y reversibilidad de los modos de razonar (Piaget, 1973). A nuestro entender, los bajos porcentajes de respuestas correctas at problema núm. 7 revelan la incapacidad de los estudiantes para razonar holísticamente. El problema núm. 6 sólo desencadena reacciones automatizadas y estereotipadas, habituales en la enseñanza centrada en aspectos algorítmicos y en la manipulación de reglas y ecuaciones.

\section{H3. Razonamiento puramente algorítmico}

Este tipo de razonamiento aparece en las respuestas de los estudiantes a las situaciones planteadas en los enunciados $8,9,10$ y 11 del apéndice.

En el problema núm. 8 , de los 17 estudiantes, sólo 1 responde correctamente que la ley de Ohm estabtece la proporcionalidad directa entre la caída de tensión y la intensidad de la corriente eléctrica sobre un conductor, a través de una constante (Ilamada resistencia) cuyo valor depende del material y de la temperatura (no menciona la incidencia de parámetros geométricos). Otros 6 alumnos dicen que la ley de Ohm establece una relación lineal entre la caída de tensión y la intensidad de la corriente eléctrica sobre un conductor a través de una constante (la resistencia). Estas últimas respuestas, si bien son esencialmente correctas, no tienen la profundidad de la brindada por el primer alumno mencionado.

Los otros 10 estudiantes señalan que la ley de Ohm:

- establece que la resistencia de un conductor es directamente proporcional a $\mathrm{V}$ e inversamente proporcional a $\mathrm{I}(\mathrm{N}=4)$;

- establece que la intensidad de la corriente es inversamente proporcional a $\mathrm{V}$ e inversamente proporcional a $\mathrm{R}$ $(\mathrm{N}=4)$;

- permite calcular la tensión sobre un conductor multiplícando entre sí los valores de $\mathrm{R}$ e $\mathrm{I}(\mathrm{N}=2)$.

Es decir, que casi el $60 \%$ de los estudiantes enuncia la ley de Ohm «leyendo la formula» V = I .R (o alguna otra relación đerivada de ésta), y no señala el aspecto crucial de la independencia del valor de la resistencia $R$ con la diferencia de potencial aplicada y con la intensidad de la corriente que circula. 
En el problema núm. 9, que fue presentado a una muestra de estudiantes diferente a la que resolvió el problema núm. 8, el $55 \%$ de los alumnos atribuyó a la resistencia $\mathrm{R}$ de un conductor ohmico, dependencia con la tensión eléctrica aplicada, o con la intensidad de la corriente que circula, o con el campo eléctrico, o con la densidad de corriente, mediante un tratamiento puramente algebraico de lats relaciones $R=V / I ; \rho=E / J$. Este tratamiento es, además, físicamente incorrecto, pues implica suponer que $\mathrm{V}$ con $\mathrm{I}$, y $\mathrm{E}$ con $\mathrm{J}$, son independientes entre sí.

El $25 \%$ de los estudiantes afirma que el valor de $R$ no depende de la forma dada al conductor, sino sólo de su longitud y su sección de acuerdo con la relación $\mathrm{R}=\rho . \mathrm{I} / \mathrm{S}$.

Esta última relación es empleada por el $100 \%$ de los estudiantes para justificar la dependencia de $R$ con eI material y las dimensiones geométricas. Ningún estudiante da razones físicas, conceptuales, ni otorga importancia al hecho de que ésta no es una expresión para la resistencia de un conductor cualquiera, sino que sólo es aplicable a conductores cilíndricos largos, de sección transversal $\mathrm{S}$ y de longitud I.

En el problema núm. 10, el $40 \%$ de los estudiantes tiene dificultades para advertir que los circuitos I y II son equivalentes. EI $50 \%$ asigna incorrectamente los diagramas (i) o (iii) al circuito Il.

En el problema núm. 11 , sólo el $15 \%$ de los estudiantes identifica el diagrama (iii) como el esquema correspondiente a un puente de Wheatstone en equilibrio. El $75 \%$ acepta el esquema (i) como la representación correcta.

Tanto en el problema núm. 10, como en el núm. I1, que fueron respondidos por diferentes muestras de alumnos, aparece la dificultad de los estudiantes para abstraer un diagrama de elementos eléctricos y conexiones a partir de un circuito real. En nuestra práctica docente en un laboratorio para estudiantes de ingeniería y de licenciatura en física, hemos enfrentado reiteradamente también la dificultad de los estudiantes para resolver la situación inversa: dado un esquema, armar el circuito real correspondiente. Muchos estudiantes atribuyen importancia a las relaciones espaciales, a la ubicación (en cuadrado, en triángulo, etc.) de los elementos y a uniones de conductores que no implican ninguna modificación en el comportamiento del circuito.

\section{H4. Razonamiento reduccionista}

Este tipo de razonamiento aparece en las respuestas de los estudiantes a las situaciones planteadas en los enunciados $12,12^{\prime}$ y 13 del apéndice.

En el problema núm. 12, de Ios 16 estudiantes, 6 responden correctamente, señalando su desacuerdo con la afirmación y explicando que un amperímetro se conecta en serie porque de esa manera puede cumplir con su objetivo de medir la intensidad de corriente eléctrica.
Los otros 10 estudiantes (más del $60 \%$ de la muestra) están de acuerdo con la afirmación. En las justificaciones, todos ellos mencionan que un amperímetro con una resistencia interna pequeña introduce menor error en la medición de una resistencia comparado con otro amperímetro de mayor resistencia interna.

En el problema núm. 12 (que fue suministrado a una muestra diferente de estudiantes), se repite este tipo de razonamiento, pero los porcentaje de respuestas incorrectas son mayores. Sólo 2 alumnos responđen correctamente. El resto (casi el $90 \%$ de la muestra) está de acuerdo con la afirmación y lo justifica en términos del error de interacción introducido por la resistencia interna del voltímetro al medir el valor de una resistencia $\mathbf{R}$.

Tanto en las respuestas del problema núm. 12, como en las del núm. 12', se advierte en los estudiantes una confusión entre la finalidad perseguida con el uso de los instrumentos (sus funciones) y las formas ideadas para disminuir la interacción entre lo que se mide y lo que es medido (sus propiedades).

En el problema núm. 13, de los 27 estudiantes, 6 responden correctamente, prediciendo que, al conectarlos en serie, el foco que emitirá mayor intensidad luminosa será el que tiene la inscripción « $220 \mathrm{~V}, 25 \mathrm{~W}$ ». Explican de diversas maneras que en una conexión en paralelo la potencia es máxima para el foco de menor resistencia, mientras que para una conexión en serie la potencia es máxima para el foco de mayor resistencia.

De los 19 estudiantes restantes, 3 no responden y 16 (casi el $60 \%$ de la muestra total para este problema) predicen que en serie brillará más que el foco donde se lee « 220 $\mathrm{V}, 100 \mathrm{~W}$. Sus justificaciones mencionan reiteradamente el valor «I00 W» comparándolo con «40 W» y con « $25 \mathrm{~W}$, sin prestar atención al dato « $220 \mathrm{~V} »$. Atribuyen al dato "potencia» (una propiedad del foco) un carácter absoluto, independiente de la conexión en que se intercale al foco (es decir, de la función que éste desempeñe).

\section{H5. Aprendizaje insatisfactorio}

Los resultados presentados para las hipótesis $\mathrm{H} 1, \mathrm{H} 2, \mathrm{H} 3$ y $\mathrm{H} 4$ muestran un elevado porcentaje de estudiantes con aprendizaje insatisfactorio.

En todos los casos en que Ios estudiantes emplearon modos de razonamiento no científico, sus respuestas fueron discrepantes con las elaboradas para esas mismas situaciones por la comunidad cientifica.

Esta afirmación es válida incluso para aquellos casos en que la conclusión fínal coincidía accidentalmente con la científica, pero estaba basada en un razonamiento no científico que en otros problemas producía respuestas finales discrepantes. Debe tenerse claro que el conocimiento científico no es meramente descriptivo, sino explicativo, coherente y sistémico, por lo que no puede aceptarse como legítima una respuesta basada en un 
modo de razonar que aigunas veces conduce a una respuesta correcta, y otras veces a una respuesta incorrecta, sin que medie ningún cambio en el paradigma o modelo teórico de referencia.

\section{CONCLUSIONES}

A lo largo de la historia de la que somos herederos culturalmente, la humanidad ha desarrollado (y desarroIIa) diferentes tipos de saberes sobre el mundo físico, en respuesta a diferentes motivaciones, metas, valores prevalecientes, conocimientos previos, criterios para juzgar la validez del conocimiento, etc.

La mayor o menor pertinencia de los distintos enfoques frente a una situación concreta depende de la finalidad perseguida por el usuario.

Para las necesidades de la vida cotidiana, el conocimiento común, basado en los modos espontáneos de razonar, es útil y muchas veces es suficiente.

En este trabajo hemos hipotetizado sobre la existencia (en alumnos de ciclos básicos de carreras científicas) de cuatro tipos de razonamiento espontáneo incompatibles con el conocimiento científico; sobre sus características generales, y sobre su incidencia en la construcción del conocimiento relacionado con el funcionamiento de circuitos eléctricos sencillos. Se trata de los razonamientos «monoconceptual», «no sistémico, irreversible», "puramente algorftmico» $\mathrm{y}$ «reduccionista».

Los resultados obtenidos parecen validar la hipótesis de que estos tipos de razonamiento espontáneo son inadecuados para un aprendizaje significativo de ciertos contenidos de física en ciclos básicos universitarios.

A la luz de estos resultados, y en el marco de un modelo constructivista de aprendizaje, creemos que el aprendizaje de la física debe ser planteado a Ios estudiantes de ciclos básicos de carreras científicas como la adquisición de otra manera de conocer: la manera de conocer de la física clásica.

Es conveniente, a nuestro entender, un cierto proceso de metacognición por parte de los estudiantes (Hewson y Thorley, 1989) que favorezca que ellos conozcan y valoricen las metas cognitivas de la empresa científica, sus modos de razonar, sus metodologías para la construcción y control del conocimiento, sus exigencias epistemológicas.

Incluso bajo esas condiciones, parece ilusorio esperar que la manera de conocer empleado por la física moderna clásica, que tanto esfuerzo le costó alcanzar a la humanidad, sea aprendido por los estudiantes a corto plazo o como una súbita «revelación» esclarecedora. Cambios tan profundos no son alcanzados de una vez.

Los profesores podemos favorecer ese proceso diseñando e implementando actividades educativas que requieran: una participación mental activa de los estudiantes; una profundización en los significados; tratamientos cualitativos que otorguen contenido a los algoritmos y validez a la precisión cuantitativa; debates donde se confronten diversas alternativas y se tomen decisiones basándose en criterios compatibles con los científicos; consideración de situaciones diferentes para controlar la consistencia del conocimiento; etc. En síntesis, actividades de construcción de conocimiento científico a través de modos científicos de enfrentar los problemas, que no centren las cuestiones sólo en el «cambio conceptual». A nuestro criterio, es necesario atender debidamente la circunstancia de que este cambio no será significativo si no va acompañado de otros, tales como cambios en los modos de razonar al que nos referimos en este trabajo.

Así, el proceso de enseñanza y aprendizaje institucional no sólo favorecerá la transición desde sistemas cognoscitivos espontáneos hacia sistemas más científicos, sino que facilitará el desarrollo en los estudiantes de modos de razonar, de habilidades y estrategias necesarias para producir los nuevos cambios que requerirán el aprendizaje de nuevas teorías (por ejemplo, las de la física del siglo $\mathrm{XX}$ ) y su futura labor profesional como ingenieros, como físicos, como profesores e investigadores (Cudmani, Salinas y Pesa, 1991).

\section{NOTA}

Este trabajo ha sido presentado en la Octava Reunión Nacional de Educación en la Física (REF VIII) (Rosario, Argentina, 18 al 22 de octubre de 1993) y ha sido pablicado en las memorias de dicha reunión. 


\section{REFERENCIAS BIBLIOGRÁFICAS}

BACHEIARD, G. (1972). La formación del espíritu científico. Buenos Aires: Ed. Siglo XXI.

BUNGE, M. (1985). La investigación científica. Barcelona: Ed. Atiel.

CARRASCOSA, J. y GIL, D. (1985). La «metodologia de la superficialitat» i l'aprenentatge de les ciències. Enseñanza de las Ciencias, 3(2), pp. 113-120.

CLOSSET, I.L. (1983). Le raisonnement sequentiel en electrocinetique. Tesis doctoral. Labotatoire de Didactique de la Physique dans I'Enseignement Supérieur, Université Paris VII.

CUDMANI, L.C. DE, SALINAS DE SANDOVAL, I. y PESA DE DANÓN, M. (1991). La generación autónoma de «conflictos cognoscitivos» para favorecer cambios de paradigmas en el aprendizaje de la física. Enseñanza de las Ciencias, $9(3)$.

GIL, D. (1983). Tres paradigmas básicos en la enseñanza de las ciencias. Enseñanza de las Ciencias, 1(1), pp. 26-33.

GIL, D. y CARRASCOSA, J. (1985). Science learning as a conceptual and methodological change. European Journal of Science Education, 7(3), pp. 231-236.

HEMPEL, C. (1987). Filosofía de la ciencia natural. Madrid: Alianza Editoriat.

HEWSON, P.W. y THORLEY, R. (1989). The condition of conceptual change in the classroom. International Journal of Science Education, 11(5), pp. 541-553.

HODSON, D. (1986). Philosophy of Science and Science of Education. Journal of Philosophy of Education, 20(2).
MANRIQUE, M.J., VARELO P. y FAVIERES, A. (1989). Selecciones bibliográficas temáticas: Selección sobre esquemas alternativos de los estudiantes en electricidad. Enseñanza de las Ciencias, 7 (3), pp. 292-295.

MATTHEWS, M.R. (1992). History, Philosophy and Science Teaching: The Present Rapprochement. Science and Education, 1(1), pp. $1-47$.

MCDERMOTT, L.C. y SHAFFER, P.S. (1992), Research as a guide for curriculum development: An Example from Introductory Electricity - Parts I and II. Am. J. Phys. 60(11), pp. 994-1013.

PIAGET, J. (1972). Psicología y epistemología. Buenos Aires: Emecé Editores.

PIAGET, J. (1973). Psicología de la inteligencia. Buenos Aires: Ed. Psique.

PIAGET, J. y GARCÍA, R. (1982). Historia y sicogénesis de la física. Buenos Aires: Ed. Siglo XXI.

POPPER, K. (1985). La lógica de la investigación científica. Madrid: Ed. Tecnos.

SALINAS DE SANDOVAL, J. y CUDMANI, L.C, DE (1993). Razonamientos espontáneos en circuitos eléctricos sencilios: transferencia de resultados de investigación educativa a un laboratorio universitario básico. (En prensa).

VIENNOT, L. (1983). Implicit Statements in Physics: students and constants, Proceedings of the International Seminar. Misconceptions in the Science and Mathematics, pp. 133136. Cornell University.

VILLANI, A. y PACCA, J. (1990). Spontaneous reasoning of graduate Students. International Journal of Science Education, 12(5), pp. 589-600.

[Artf́culo recibido en septiembre de 1993 y aceptado en marzo de 1996.] 
APÉNDICE 1

CUESTIONES PLANTEADAS A LOS ESTUDIANTES

Problema núm. $1: I(N=20)$

(Enunciado adaptado de Viennot, 1983.)

En el circuito de la figura, dos resistencias $R_{1}$ y $R_{2}$ están conectadas a una fuente de tensión $V_{0}$. Se aumenta el valor de $R$, y se observa que la lectura $V$ en el voltímetro también aumenta. Un alumno explica que «V = I. R1 y, como $R$, aumenta, $V$ aumenta».

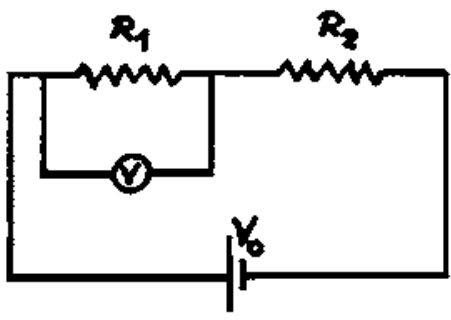

a) ¿Está usted de acuerdo con este razonamiento? (Sí/No)

b) Aclare las razones de su respuesta.

\section{Problema núm. 2: $(\mathrm{N}=20)$}

Con una pila pequeña común (de aproximadamente $0,1 \Omega$ de resistencia interna) y dos foquitos de linterna, se arma el circuito de la figura. Luego de unos segundos, se abre el circuito en el punto $S$.

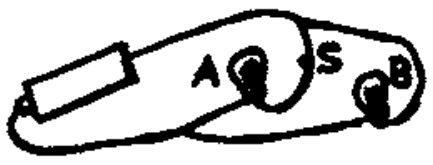

a) ¿Cambiarâ la intensidad luminosa emitida por el foco $B$ ? (Síno)

b) Justifique su respuesta.

Problema núm. 3: $(\mathrm{N}=31)$

En el circuito de la figura, todos los focos son iguales, y la fuente suministra una tensión constante $V_{0}$. Luego de unos segundos de funcionamiento, se quema el foco B.

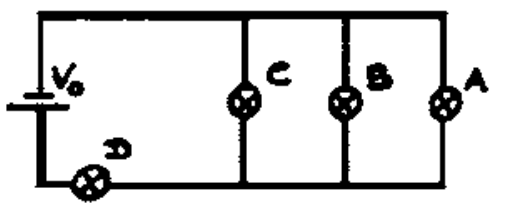

a) ¿Varía la intensidad luminosa emitida por cada uno de los otros focos? (Considérelos uno por uno).

b) Justique surs respuestas en todos los casos, sean éstas afirmativas o negativas.

Problema núm. 4: $(\mathrm{N}=20)$

Considere los circuitos de las figuras, donde $\mathrm{R}<\mathrm{R}_{\text {foco }}$

(r)

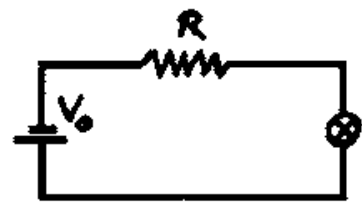

(x)

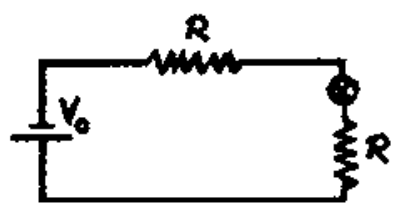

(III)

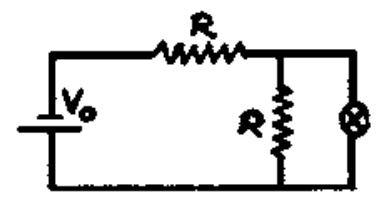

a) Complete el siguiente cuadro:

* La mfnima intensidad Iuminosa en el foco se obtiene con el circuito....

* Le sigue, con intensidad creciente, el circuito.....

* La máxima intensidad luminosa en el foco se obtiene con el circuito.....

b) Justifique sus respuestas. 
Problema num. 5: $(\mathrm{N}=23)$

En el circuito de la figura, todos los focos son iguales, y la fuente suministra una tensión constante $\mathrm{V}_{\mathrm{D}}$. Luego de unos minutos de funcionamiento, se quema el foco $\mathrm{B}$.

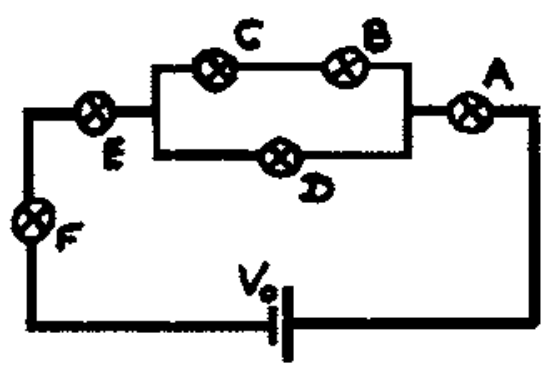

a) Varía la intensidad luminosa emitida por cađa uno de los focos? (Considérelos uno por uno).

b) Justifique sus respuestas en todos los casos, sean éstas afirmativas o negativas.

Problema núm. 6: $(\mathrm{N}=20)$

Se necesita medir una resistencia de aproximadamente $15 \Omega$. Se dispone de un amperímetro y un voltímetro con las siguientes características:

Amperímetro $\quad\left\{\begin{array}{l}\text { rango: } 0 \text { a } 2,5 \mathrm{~A} \\ 100 \text { divisiones en la escala } \\ \text { clase } 2\left(^{*}\right) \\ \text { resistencia interna } 0,2 \Omega \\ \text { Voltímetro }\end{array} \quad\left\{\begin{array}{l}50 \text { divisiones en la escala } \\ \text { clase } 1 \text { (*) } \\ \text { resistencia interna } 10 \Omega\end{array}\right.\right.$

El circuito se conectará a una fuente de tensión que suministra aproximadamente $35 \mathrm{~V}$ de tensión continua.

a) Haga un esquema del circuito que usaría. Justifique su elección.

b) Estime el error mínimo que se puede cometer al medir $\mathrm{R}$ con tales elementos y circuito.

(*) La clase da el error porcentual de exactitud a plena escala.

Problema núm. 7: $(\mathrm{N}=17)$

Se desea medir una resistencia de aproximadamente $100 \Omega$. Se dispone de una fuente de tensión que suministra unos $200 \mathrm{~V}$ de tensión continua. Se cuenta con un amperimetro de rango 0 a $\mathrm{I}$ A, clase 2 , resistencia interna $0,1 \Omega$ y 100 divisiones en la escala.

a) Seleccionte las características de un voltímetro idóneo para ser empleado en esta medición. Justifique sus criterios.

b) Haga un esquema del circuito que usaría. Justifique su eleccion.

c) Estime el error mínimo que se puede cometer al medir $R$ con tales elementos y círcuito.

Problema núm. 8: $(\mathrm{N}=I 7)$

Exprese con paiabras, no con fórmulas, el enunciado de la ley de Ohm en la forma más clara y completa posible.

Problema núm. 9: $(\mathrm{N}=20)$

Sea R la resistencia de un conductor ómmico.

$\mathrm{El}$ valor de $\mathrm{R}$ depende de: 
a) la tensión eléctrica apiicada

b) la corriente que circula

c) el campo eléctrico aplicado

d) la forma deI conductor

e) la densidad de la corriente

f) el material con que está construido

g) las dimensiones geométricas del conductor

Justifique todas sus respuestas, sean afirmativas o negativas.

Problema núm. 10: $(\mathrm{N}=22)$

(Enunciado propuesto por McDermott y Shaffer, 1992).

Indique las correspondencias entre los circuitos I y II y los diagramas $\mathrm{i}$, ii, iii y iv.

a) ¿Qué diagrama corresponde al circuito I?

Justifique la respuesta.

b) ¿Qué diagrama corresponde al circuito II?

Justifique la respuesta.
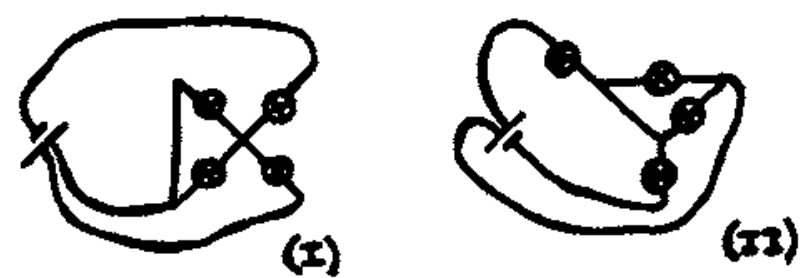

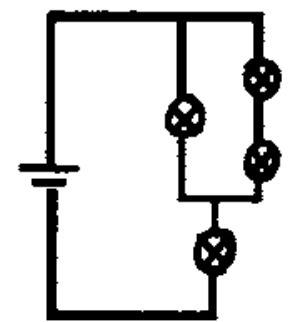

(i)

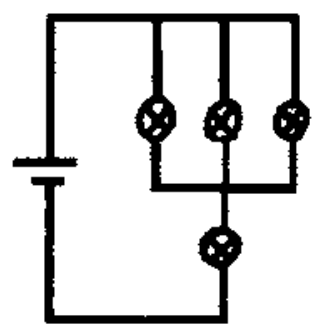

(ii)

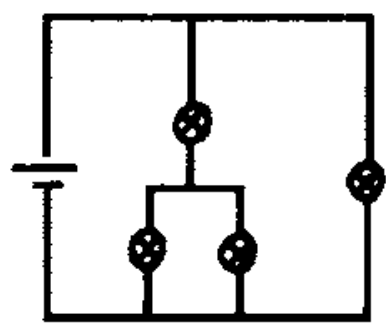

(iii)

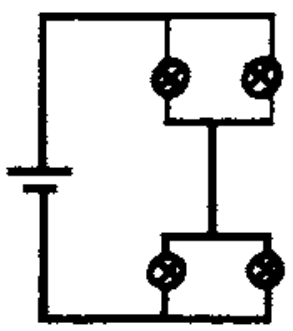

(iv)

Problema núm. $11:(\mathrm{N}=20)$

¿Corresponde/n alguno/s de los siguientes diagramas al esquema de un puente de Wheatstone en equilibrio? Caso afirmativo, ¿cuál es y por qué? Caso negativo, ¿por qué no corresponde/n?
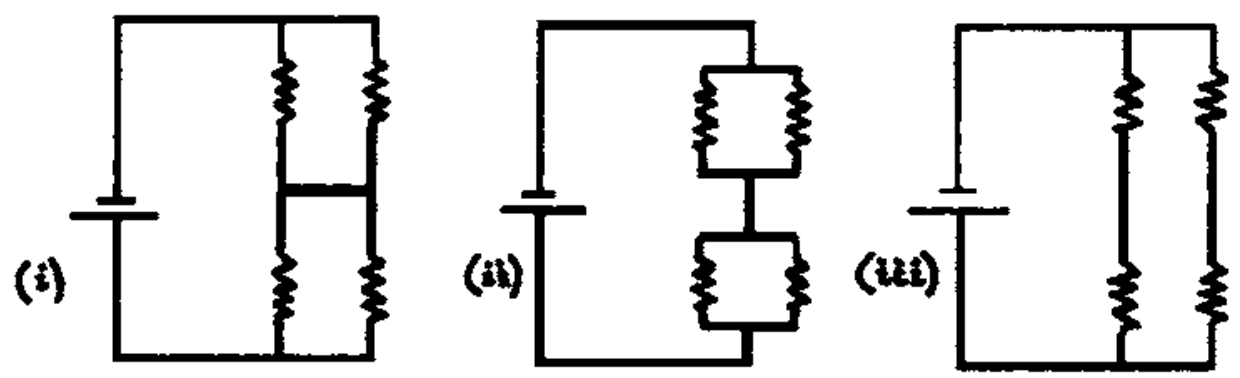
Problema núm. 12: $(\mathrm{N}=16)$

Un alumno afirma que «los amperímetros se conectan en serie porque tienen resistencias internas pequeñas». ¿Está usted de acuerdo con esta afirmación? ¿Por qué?

Problema núm. 12': ( $\mathrm{N}=18)$

Un alumno afirma que «los voltímetros se conectan en paralelo porque tienen resistencias internas elevadas». ¿Está usted de acuerdo con esta afirmación? Por qué?

Problema núm. 13: $(\mathrm{N}=27)$

Tres focos donde se lee, respectivamente, « $220 \mathrm{~V}, 25 \mathrm{~W} », « 220 \mathrm{~V}, 40 \mathrm{~W} » \mathrm{y} « 220 \mathrm{~V}, 100 \mathrm{~W} »$ se conectan en serie a la red domiciliaria de $220 \mathrm{~V}$. ¿Cuál de ellos emitirá mayor intensidad Iuminosa? ¿Por qué? 